\title{
Getting Rid of Covid-19 Symptoms Quickly: A Case Report
}

\author{
Mohammad Yavari $^{1}$, Ghazal Esmaeilimoakhar ${ }^{2}$ and Hossein Esmaeili ${ }^{3 *}$ \\ ${ }^{1}$ Bachelor of Science in Nursing, Islamic Azad University Tehran Branch, Tehran, Iran \\ ${ }^{2}$ Medical Student, Young Researcher and Elite Club, Tehran Medical Sciences, Islamic Azad University, Tehran, Iran \\ ${ }^{3}$ Young Researcher and Elite Club, Tehran Medical Sciences, Islamic Azad University, Tehran, Iran
}

Submission: January 22, 2020; Published: February 03, 2021

"Corresponding author: Hossein Esmaeili, Young Researcher and Elite Club, Tehran Medical Sciences, Islamic Azad University, Tehran, Iran

\section{Abstract}

Background: Nowadays, a variety of substances are used for Covid-19 prevention or treatment and probably, no effective and reliable treatment has been discovered yet. In this case report, we considered the possibility of some mineral and herbal substances' effectiveness on a patient who has a background of different diseases (as important risk factors of Covid-19 infection).

Case: This report is about a 56-year-old man Who was infected with Covid-19. This man has a history of DM, BP and because of being exposed to chemical agents in war, he always has a high level of $\mathrm{HbA1C}$. The patient has symptoms such as right-side paresis, left side numbness, shortness of breath, severe pain in sternum referred to his back, pain in heart, anorexia, dry cough, severe body trembling and feeling body inner heat without having fever.

Conclusion: A combination of lemon, black pepper and salt can be effective in decreasing cough and chest pain and a combination of ginger rose-water, garlic and mint-water can be effective in decreasing shortness of breath, pain and anorexia. According to the results of this article it seems most likely that the second combination, ginger, rose-water, garlic and mint-water can also be effective in prevention of Corona symptoms. This report can be the basis for further clinical research on the effectiveness of these substances in Corona treatment.

Keywords: Covid-19; Garlic; Zinger; Lemon; Pain, Chemical agents

Abbreviations: DM: Diabetes mellitus; BP: Blood Pressure; HbA1C: Hemoglobin A1c; BS: Blood Sugar

\section{Case presentation}

This report is about a 56-year-old man who was infected with Covid-19 (Figure 1). This man has a history of DM, BP and because of being exposed to chemical agents in war, he always has a high level of $\mathrm{HbA1C}$. This man lives with his wife and daughter. Without considering any of suggested health protocols, they have been in direct contact with each other. Better to be mentioned, BP [1], DM [2] and high level of HbA1C [3] are risk factors leading to acute Corona disease.

At the very beginning, the patient attended a clinic semiconsciously, suffering from hallucination, severeheadache, and delusion. BS:336 and BP:170/120 (systole:170 and diastole:120) was reported and according to the doctor's prescription, he had a Furosemide injection. The day after attending the clinic, the patient was hospitalized at home while he had new symptoms, such as right-side paresis, left side numbness, shortness of breath, severe pain in sternum referred to his back, pain in heart, anorexia, severe body trembling and feeling body inner heat without having fever. Despite the appearance of new symptoms in the patient, the family members still don't pay attention to health protocols and keep having direct contact with the patient. The patient's wife starts to give the patient some herbal and mineral nutrients in a regular routine. After undergoing a CT Brain, no brain abnormality was detected in the patient (Figure 2).

\section{Disscusion}

From the appearance of the symptoms, it took only 3 days for the symptoms (except numbness) to disappear.

\section{Day 1:}

The patient had all the symptoms mentioned. His wife gave him some rose and ginger decoction with 3 garlics and mint-water 3 times a day in the morning, at noon and in the evening. (he used 2 glasses in the morning, 1 glass at noon and 1 glass in the evening.) 


\section{Novel Approaches in Drug Designing \& Development}

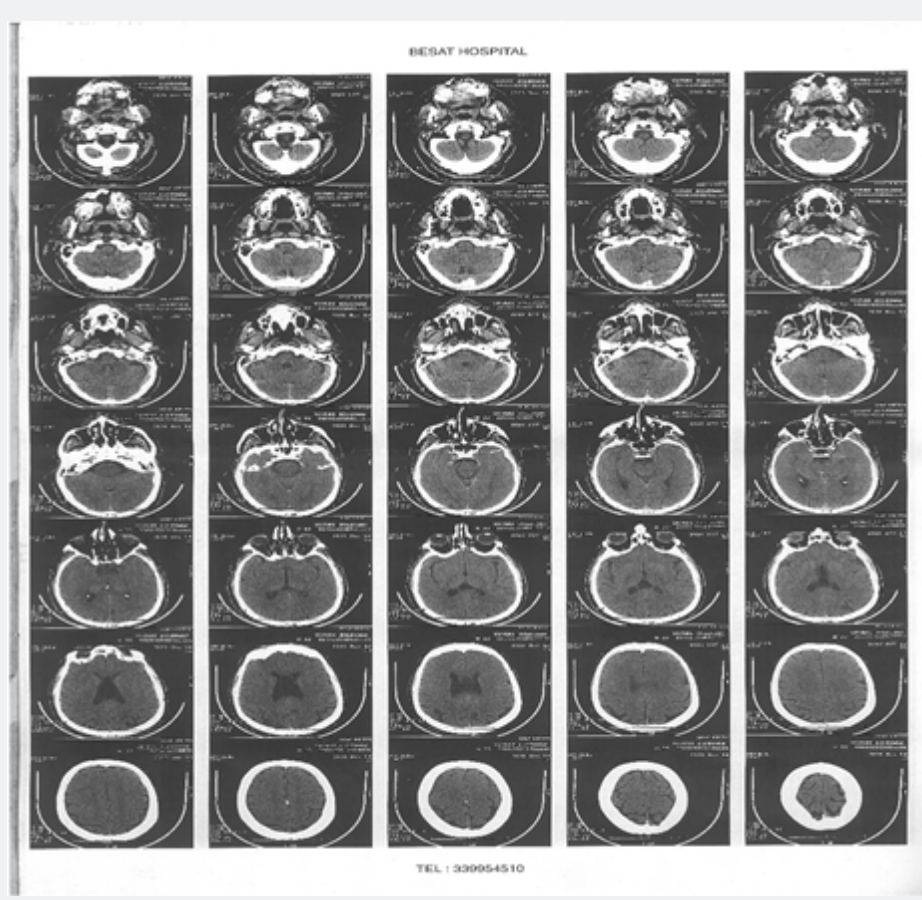

Figure 1: patient Lung MRI with Covid-19.

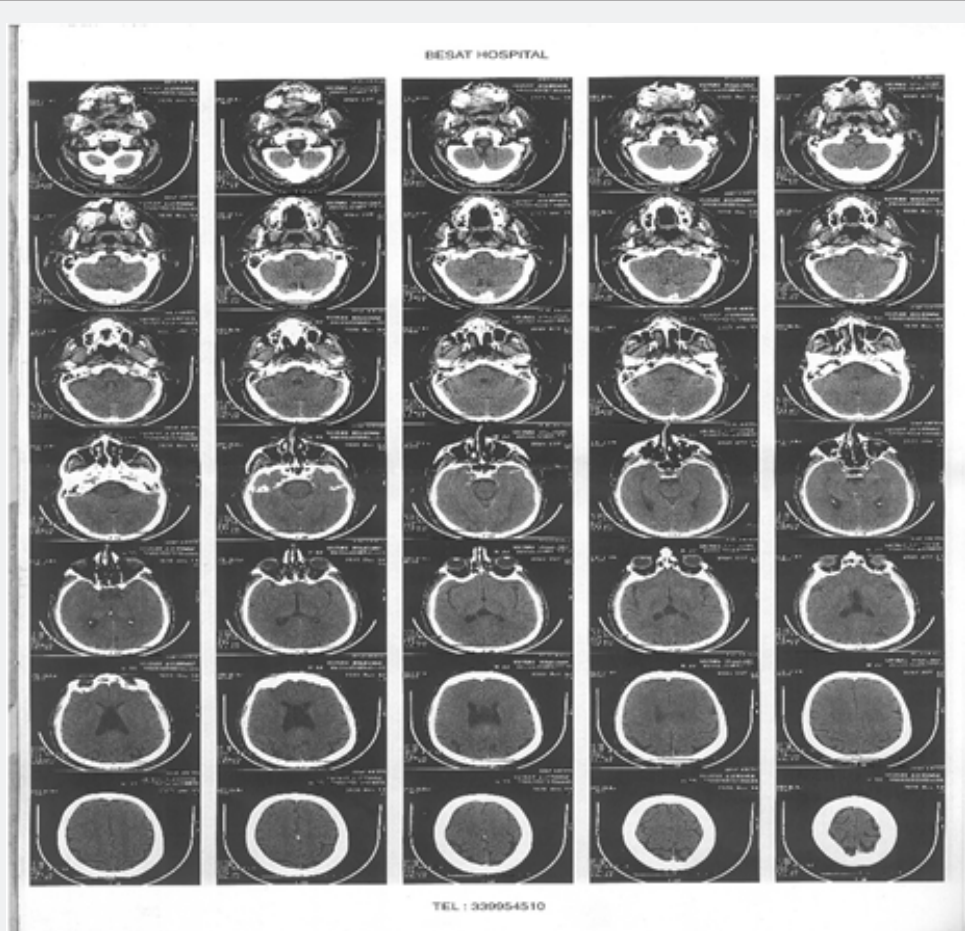

Figure 2: The patient's brain image does not show any problems.

\section{Day 2:}

All of the symptoms were relieved except body numbness. In the other hand, dry cough was added to the symptoms. The patient's wife continued to give first day's nutrients to the patients and in order to treat cough, she gave him 5 lemons dipped in black pepper and salt. The patient's cough decreased a lot by the end of the day.

\section{Day 3:}

After continuing usage of the nutrients in the first 2 days, symptoms such as paresis, shortness of breath, dry cough, 
anorexia, body trembling, feeling of inner heat and chest pain faded away completely.

The patient's wife used the same nutrients she gave to the patient herself from the first day and as a result, she didn't experience any of the symptoms. But their daughter refused to use these nutrients and consequently, she had the symptoms in a while. However, after using the nutritious herbal and mineral substances, her pulmonary symptoms such as dry cough were relieved in 3 days, which reveals the effectiveness of these substances in prevention and treatment of Corona pulmonary symptoms.

Some researchers have proved that garlic $[4,5]$, ginger $[6,7]$ and lemon [8] can be effective in prevention and treatment of Corona disease.

\section{Acknowledgements}

Special thanks to the patient's family for their satisfaction and participation in this publication..

\section{References}

1. Huang I, Lim MA, Pranata R (2020) Diabetes mellitus is associated with increased mortality and severity of disease in COVID-19 pneumonia-a systematic review, meta-analysis, and meta-regression. Diabetes \& Metabolic Syndrome: Clinical Research \& Reviews 14(4): 395-403.
2. Cook TM (2020) The importance of hypertension as a risk factor for severe illness and mortality in COVID-19. Anaesthesia 75(7): 976-977.

3. Merzon E, Green I, Shpigelman M, Vinker S, Raz I, et al. (2020) Haemoglobin A1c is a predictor of COVID-19 severity in patients with diabetes. Diabetes/metabolism research and reviews: e3398.

4. Pawar YD, Patil A (2020) Garlic: An Immunity Booster Spice during Pandemic Situation. Biotica Research Today 2(9): 978-80.

5. Thota SM, Balan V, Sivaramakrishnan V (2020) Natural products as home-based prophylactic and symptom management agents in the setting of COVID-19. Phytotherapy Research 34(12): 3148-3167.

6. Ohanube GA, Obeta MU, Ikeagwulonu RC, Jwanse IR (2020) COVID-19: A Case Study of Using Vitamin C Enriched Plants and Ascorbic Acid as Cure. Am J Med Case Rep 8(11): 435-437.

7. Magzoub M (2020) Lifestyle Guideline of Ginger (Zingiber officinale) as Prophylaxis and Treatment for Coronaviruses (SARS-CoV-2) Infection (COVID-19). Saudi Journal of Biomedical Research 5(6): 125-127.

8. Runde M (2020) Validation of Perception of Some Nigerians on the Origin and Use of Phyto-remedies in Management of Covid 19; An Overview of Social Media Respondents. Communication in Physical Sciences 6(1).
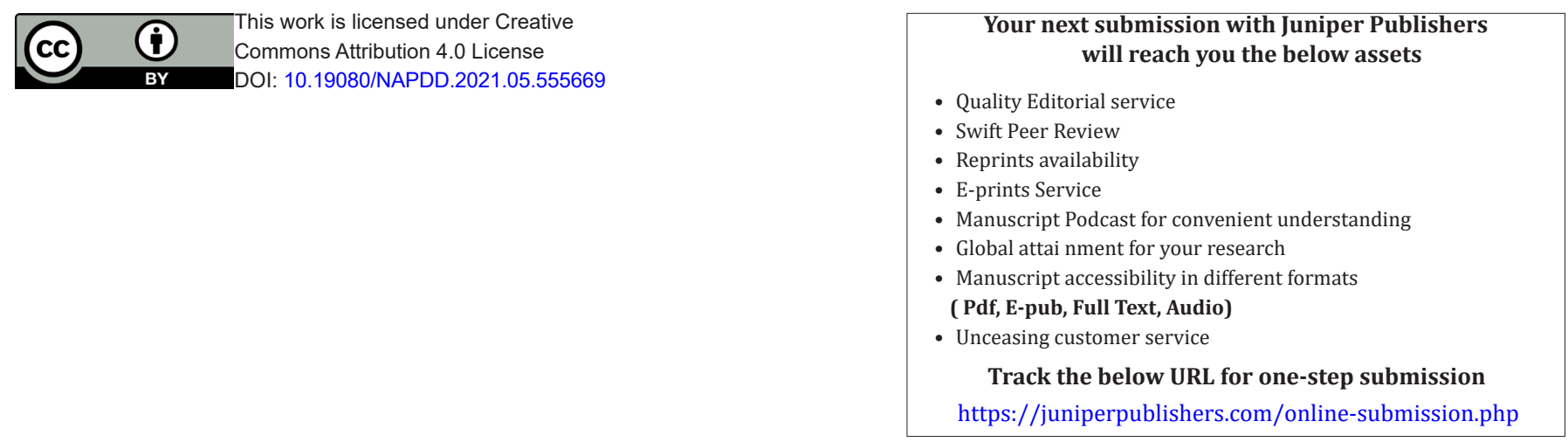